\title{
Quantum interference Hall effect in nanopatterned two-dimensional electron gas systems
}

\author{
Hang Xie and Ping Sheng \\ Department of Physics, The Hong Kong University of Science and Technology, Clear Water Bay, Kowloon, Hong Kong
}

(Received 28 November 2008; revised manuscript received 4 March 2009; published 8 April 2009)

\begin{abstract}
We show that in mesoscopic two-dimensional electron gas systems, quantum interference caused by predesigned nanopatterns can enhance the Hall effect by up to $500 \%$. A quality factor $Q$ is defined which optimizes the ratio of the Hall voltage to longitudinal voltage. Genetic algorithm and the Landauer-Büttiker formalism were used to search for the potential configuration that can achieve a large $Q$. We propose some realistic nanopatterns to realize this effect.
\end{abstract}

DOI: 10.1103/PhysRevB.79.165307

PACS number(s): 72.20.My

\section{INTRODUCTION}

Quantum Hall effect shows that electron's quantum wave nature can alter its classical Hall character under a strong magnetic field. ${ }^{1}$ But can such wave characteristics persist in the limit of $B \rightarrow 0$ ? Some time ago it was shown that the giant Hall effect (GHE) in magnetic materials can persist even in the limit where magnetization has reached saturation and therefore cannot play a role. ${ }^{2}$ It follows that there could be some alternative scenario for the enhancement of the Hall effect that are distinct from the usual GHE.,3 Motivated by this finding, subsequent experimental and theoretical studies on the nonmagnetic $\mathrm{CuSiO}_{2}$ composites have found that quantum interference may indeed play a crucial role in enhancing the Hall coefficient. ${ }^{4,5}$ This conclusion was supported by the fact that only when the size of the metallic granules is much smaller than the coherent length of the electron, e.g., in the low-temperature regime (so that the electron coherence length can reach its saturation value), can this GHE occur. Most dramatically, the effect vanishes in annealed samples, when the size of the metallic granules exceeds the quantum coherence length. Together with the fact that all the GHE was observed in the vicinity of the percolation threshold, it was concluded that the GHE arises from the quantum interference effect in the percolation geometric setting. By using mesoscopic transport calculations in the microscopic regions (within a coherence length) to evaluate the local Hall conductances, Wan and Sheng ${ }^{5}$ built a model in which the local (mesoscopic) Hall conductance tensors thus obtained served as the inputs to the macroscopic classical electric network calculations. The results show excellent agreement with the experiment data.

The random nature of the composite geometry means that the GHE is manifest only in a statistically averaged sense. In this paper, we show in mesoscopic two-dimensional (2D) electron gas systems one can utilize predesigned nanostructures to maximize the Hall effect in the limit of small magnetic field. Genetic algorithm was used to search the optimal 2D structure pattern. It is shown that for certain nanopatterned configurations the Hall coefficient can be enhanced up to $300 \%-500 \%$ in 2D electron systems (with parameters relevant to semiconductor heterostructures). By using designed nanostructures, the case for quantum interference Hall effect can be much better verified, as there can be a direct correlation between the nanostructure and the measured Hall characteristics.

In what follows, the Hall effect in the mesoscopic context, i.e., in nanostructures, is described in Sec. II together with its calculational approach. In Sec. III we present the results on the Hall effect in homogeneous mesoscopic samples in order to set the stage for the consideration of patterned samples. In Sec. IV the definition of a quality factor for measuring the mesoscopic Hall effect is followed by pattern optimizations to achieve maximum Hall-effect enhancement. A few optimal nanopatterns are presented and their relevant parameter values compared with those of the homogeneous sample(s).

\section{MESOSCOPIC HALL EFFECT}

Our 2D electron gas system can be a semiconductor heterostructure, with a Fermi wavelength comparable to the size of the nanopattern to be considered below. We would like to evaluate the electronic transport behavior under crossed electric and magnetic fields in which the electron's wave characteristics are explicitly taken into account. For simplicity only the square sample geometry will be considered, with four sides connected to the four terminals as shown in Fig. 1. In the following we first describe the calculation of the transmission tensor $\left(T_{p q}\right)$ between the terminals $p$ and $q$. The Landauer-Büttiker formula ${ }^{6}$ will be used to obtain sample's conductance tensor.

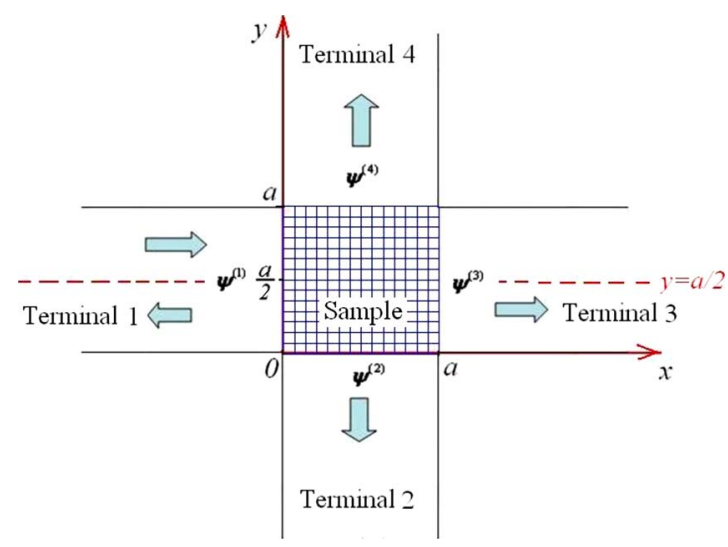

FIG. 1. (Color online) Geometry and the sample grid for the four-terminal calculation. 


\section{A. $T_{i j}$ calculation in the four-terminal configuration}

For the four-terminal system, the transmission coefficient can be calculated by the mixed boundary matching method in which the wave functions in the electrical terminal regions are expressed as the superposition of (waveguide) eigenmodes, and in the sample region by using the discrete basis (defined at each grid node). ${ }^{7}$ The values at the grid nodes and the expansion coefficients are the unknowns. The relevant equations are of two types: one is from the discretized Schrödinger equation for each grid node, and the other is from the boundary conditions at the interfaces between the sample and the terminals. Figure 1 shows the geometry and the sample grid for this four-terminal system.

The Schrödinger equation in the presence of a magnetic field is given by

$$
\frac{1}{2 m}(\vec{p}-e \vec{A})^{2} \psi(\vec{r})+V(\vec{r}) \psi(\vec{r})=E \psi(\vec{r}),
$$

where $\vec{p}=-i \hbar \vec{\nabla}$ and $\vec{A}$ denotes the vector potential. The magnetic field may be expressed as $\vec{B}=\vec{\nabla} \times \vec{A}$. Here $V(\vec{r})$ is the scalar (electric) potential. For simplicity, we set $V(\vec{r})=0$ in the terminal areas with an infinite potential step defining terminal's confining boundaries, inside which the electronic waves may be simply regarded as guided modes. In Eq. (1), $E$ is the energy, $e$ is the electron charge, and $m$ is the electron mass.

For terminals 1 and 3 , we use the Landau gauge $\vec{A}$ $=(B y, 0,0)$ together with the waveguide solution $\psi$ $=e^{i k \cdot x} \varphi(y)$ in Eq. (1). By using the separation of variables, we obtain

$$
-\frac{d^{2} \varphi(y)}{d y^{2}}+\left[(k-P \cdot y)^{2}-k_{0}^{2}\right] \varphi(y)=0,
$$

where $P=e B / \hbar$.

We denote $k(n)$ the eigenvalue and $\varphi_{n}(y)$ the eigenfunction of Eq. (2) under the boundary condition $\varphi(0)=\varphi(a)=0$, where $a$ denotes the terminal's transverse width. To distinguish the two propagation directions in a magnetic field, two sets of eigenvalues and eigenfunctions are used. In terminals 2 and 4, the wave function has the form $\psi^{(2,4)}(x, y)$ $=e^{\mp i k \cdot y} \varphi(x)$, and a similar procedure is used to obtain the eigenfunctions by using another gauge for the purpose of variable separation. To ensure that the unitary gauge condition is satisfied for the vector potential $\vec{A}$ throughout the system, $\varphi_{n}(x)$ must differ from $\varphi_{n}(y)$ with an additional (gauge) transformation factor $\exp ($ PPxy) (see Appendix A). Hence the eigenfunctions in terminal 2 and 4 are denoted with two variables, e.g., $\varphi_{n}^{\prime}(x, y)$. The details are given in Appendix A. Here we write down the wave functions in the terminal areas as

$$
\begin{gathered}
\psi^{(1)}=e^{i k_{r}\left(n_{0}\right) \cdot x} \varphi_{n_{0}}(y)+\sum_{n=1}^{N_{1}} A_{n} e^{-i k_{f}(n) \cdot x} \varphi_{\bar{n}}(y), \\
\psi^{(3)}=\sum_{n=1}^{N_{1}} B_{n} e^{i k_{r}(n) \cdot(x-a)} \varphi_{n}(y)
\end{gathered}
$$

$$
\begin{gathered}
\psi^{(2)}=\sum_{n=1}^{N_{1}} C_{n} e^{-i k_{r}(n) \cdot y} \varphi_{\bar{n}}^{\prime}(x, y), \\
\psi^{(4)}=\sum_{n=1}^{N_{1}} D_{n} e^{i k_{f}(n) \cdot(y-a)} \varphi_{n}^{\prime}(x, y),
\end{gathered}
$$

where $k_{r}(n)$ and $k_{f}(n)$ stand for the rightward (downward) and leftward (upward) eigenwavevectors, $\varphi_{n}(y)$ and $\varphi_{\bar{n}}(y)$ are the eigenfunctions for rightward and leftward waves in terminals 1 and 3 , respectively, and $\varphi_{n}^{\prime}(x, y)$ and $\varphi_{\bar{n}}^{\prime}(x, y)$ are the eigenfunctions for the upward and downward waves in terminals 4 and 2, respectively. $\left\{A_{n}\right\},\left\{B_{n}\right\},\left\{C_{n}\right\}$ and $\left\{D_{n}\right\}$ are the expansion coefficients. Equation (3a) expresses the incident wave in terminal 1 , consisting of one single eigenmode with mode index $n_{0}$ and the reflected wave as the superposition of different eigenmodes with the expansion coefficients $\left\{A_{n}\right\}$. Here $N_{1}$ is total number of eigenmodes. From the discrete sine transformation, we set $N_{1}=N_{a}-2$, which equals the free grid nodes at the sample/terminal interface $\left(N_{a}\right.$ is the number of nodes on the grid boundary). Equations (3b)-(3d) have similar meanings.

In the middle sample area, the wave function is expressed by discrete basis defined at each grid node. With the Landau gauge $\vec{A}=(B y, 0,0)$, Eq. (1) can be written as

$$
-\frac{\hbar^{2}}{2 m}\left[\left(\partial_{x}-\frac{i e B}{\hbar} y\right)^{2}+\partial_{y}^{2}\right] \psi+V(\vec{r}) \psi=E \psi \text {. }
$$

In the sample area, we use the central difference format to discretize the Laplacian

$$
\nabla^{2} \psi_{i, j}^{S} \approx\left(\psi_{i+1, j}^{S}+\psi_{i-1, j}^{S}+\psi_{i, j+1}^{S}+\psi_{i, j-1}^{S}-4 \psi_{i, j}^{S}\right) / d^{2},
$$

where $\psi_{i, j}^{S}$ denotes the wave-function value at the node $(i, j)$. Each grid cell is a square with size $\Delta_{x}=\Delta_{y}=d$, and $d$ $=a /\left(N_{a}-1\right)$. In discretized form, Eq. (4) is given by (here we use $P_{i j}$ to stand for $\psi_{i, j}^{S}$ )

$$
\begin{aligned}
& P_{i+1, j}\left(1-I \cdot C_{m} j\right)+P_{i-1, j}\left(1+I \cdot C_{m} j\right)+P_{i, j+1}+P_{i, j-1} \\
& \quad+P_{i, j}\left(k_{0}^{2} d^{2} \varepsilon_{i, j}-4-C_{m}^{2} j^{2}\right)=0,
\end{aligned}
$$

where $C_{m}=e B d^{2} / \hbar, \varepsilon_{i, j}=1-V_{i, j} / E$, and " $I$ " denotes the imaginary unit.

For the computational grid, there are $N_{a} \times N_{a}$ nodes as shown in Fig. 1. Along the $x$ (or $y$ ) direction the node index ranges from 1 to $N_{a}$, corresponding to the points from $(0,0)$ to $(a, 0)$ or $(0, a)]$. Equation $(6)$ is used for all the nonboundary grid nodes so there are a total of $\left(N_{a}-2\right)^{2}$ independent equations in the grid area.

At the sample-terminal interfaces, we set up equations from the connection conditions, which state that the wave function and its derivative should be continuous. For example, on the boundary $x=0(0 \leq y \leq a)$ we have

$$
\begin{gathered}
\left.\psi^{(1)}\right|_{x=0}=\psi_{1, j}^{S}, \\
\left.\left(d \psi^{(1)} / d x\right)\right|_{x=0}=\left(\psi_{2, j}^{S}-\psi_{1, j}^{S}\right) / d .
\end{gathered}
$$

By substituting Eq. (3a) into Eq. (7), we get 


$$
\begin{gathered}
\varphi_{n_{0}}\left(y_{j}\right)+\sum_{n=1}^{N_{1}} A_{n} \cdot \varphi_{\bar{n}}\left(y_{j}\right)=P_{1, j}, \\
i k_{r}\left(n_{0}\right) \varphi_{n_{0}}\left(y_{j}\right)+\sum_{n=1}^{N_{1}} A_{n} \cdot\left[-i k_{f}(n)\right] \cdot \varphi_{\bar{n}}\left(y_{j}\right)=\left(P_{2, j}-P_{1, j}\right) / d .
\end{gathered}
$$

For a more precise calculation, we find that $\left(P_{2, j}-P_{1, j}\right) / d$ is the approximation of $d \psi / d x$ at $x=0$ with first-order accuracy, but it is also the approximation of $d \psi / d x$ at $x=d / 2$ with second-order accuracy. So the choice of boundary being at $x=d / 2$ would lead to better precision. Thus Eq. (8b) is modified to the form

$$
\begin{aligned}
& i k_{r}\left(n_{0}\right) \cdot e^{i k_{r}\left(n_{0}\right) \cdot d / 2} \cdot \varphi_{n_{0}}\left(y_{j}\right) \\
& \quad+\sum_{n=1}^{N_{1}} A_{n} \cdot\left[-i k_{f}(n)\right] \cdot e^{-i k_{f}(n) \cdot d / 2} \cdot \varphi_{\bar{n}}\left(y_{j}\right) \\
& =\left(P_{2, j}-P_{1, j}\right) / d .
\end{aligned}
$$

Equations (8a) and (8c) are used for every discrete node at the interface, with $2 \leq j \leq N_{a}-1$. Hence there are $2\left(N_{a}-2\right)$ such equations.

On the other three sample-terminal interfaces we can also write down these connection equations. As a result, there are $8\left(N_{a}-2\right)$ connection equations. Together with the discretized Schrödinger equation, the total number of equations is $N_{f}$ $=8\left(N_{a}-2\right)+\left(N_{a}-2\right)^{2}$. By accounting for the number of free grid nodes in the sample area, $N_{a}^{2}-4$ (except for the four corner nodes), and the number of the expansion coefficients, the total number of unknowns is $N_{x}=4 N_{1}+\left(N_{a}^{2}-4\right)=4\left(N_{a}\right.$ $-2)+\left(N_{a}^{2}-4\right)$. It is easy to see that $N_{x}=N_{f}$.

All the equations are assembled into a large matrix form. Since there are many zero elements in the matrix, the sparse matrix technique can be used. ${ }^{8}$ After solving the equations, the transmission coefficient of the system can be evaluated (see Appendix B).

Figure 2 shows two examples of the $T_{p q}$ calculations in a four-terminal system. Electron wave is incident from the left terminal 1 , scatters, and then exits through all the terminals. The wave function (real part) is drawn as contours (left) and as a three-dimensional (3D) plot (right). It can be seen that when there is no magnetic field [Fig. 2(a)], the wave function is symmetric $\left(T_{14}=T_{12}\right)$; but when a magnetic field is applied [Fig. 2(b)], the electron wave is bent downward due to the Lorentz force. It is interesting to note that in Fig. 2(b), although the wave is bent toward terminal 2, the transmission coefficients satisfy the inequality $T_{14}>T_{12}$, owing to the reflection at the right bottom corner of the sample. Because of

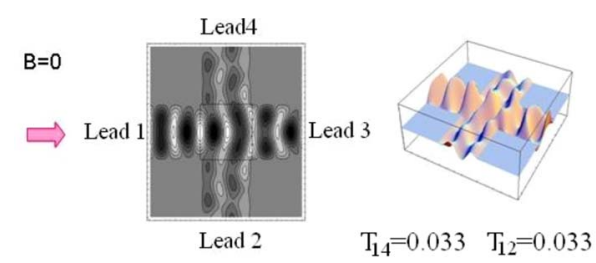

(a)

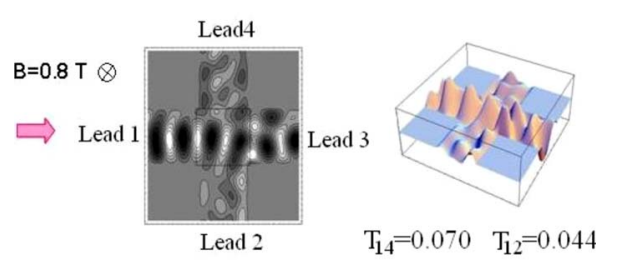

(b)

FIG. 2. (Color online) Electron wave function in a four-terminal system (a) with and (b) without the magnetic field. The real part of the wave function is drawn as a contour plot (left) and as a $3 \mathrm{D}$ plot (right). Sample size $a=40 \mathrm{~nm}$, Fermi wavelength $\lambda_{F}=20.02 \mathrm{~nm}$.

this reflection, more flux flows into terminal 4 than into terminal 2. In the calculation the normalization of the transmission (reflection) coefficients is satisfied, i.e., $T_{11}+T_{12}+T_{13}$ $+T_{14} \approx 1$, which serves as a check on the accuracy of the calculation.

\section{B. Conductance tensor evaluation}

In a multiterminal mesoscopic system, the currents and voltage in the terminals are related by the Landauer-Büttiker formula ${ }^{6}$

$$
I_{p}=\frac{2 e}{h} \sum_{q} T_{p q}\left[\mu_{p}-\mu_{q}\right]
$$

where $I_{p}$ and $\mu_{p}$ are the current and chemical potential in the terminal $p$; and $T_{p q}$ denotes the transmission coefficient from the terminal $q$ to the terminal $p$. Here $h$ is the Plank constant and $e$ is the electronic charge.

For our four-terminal system, if the sample (in the absence of a magnetic field) has a mirror-symmetry axis along the line $y=a / 2$ (see Fig. 1), then the wave function must also be symmetric so that the currents as well as their chemical potentials in terminals 2 and 4 are identical. A voltage between terminal 2 and terminal 4 occurs only in the presence of a magnetic field. We will consider only the symmetric geometries so that at zero magnetic field the Hall effect also vanishes. Below we show that the Landauer-Büttiker formula can tell us how the transmission coefficients $T_{p q}$ 's are related to the voltage at each terminal.

For simplicity, we set terminal 3 as the ground, i.e., $V_{3}$ $=0$. Then Eq. (9) can be expressed as

$$
\left(\begin{array}{c}
I_{1} \\
I_{2} \\
I_{4}
\end{array}\right)=\frac{2 e^{2}}{h}\left(\begin{array}{ccc}
T_{13}+T_{14}+T_{12} & -T_{12} & -T_{14} \\
-T_{21} & T_{23}+T_{21}+T_{24} & -T_{24} \\
-T_{41} & -T_{42} & T_{43}+T_{41}+T_{42}
\end{array}\right) \cdot\left(\begin{array}{c}
V_{1} \\
V_{2} \\
V_{4}
\end{array}\right)
$$


From Eq. (10) we obtain the relations between voltage $V_{j}$ and current $I_{j}$ as follows. Set terminal 2 and 4 as the voltage probes. That means $I_{2}=I_{4}=0$. Under this condition, the longitudinal resistance $R_{x x}\left(R_{x x}=V_{x} / I_{x}=V_{1} / I_{1}\right)$ and the Hall resistance $R_{x x}\left[R_{x y}=V_{y} / I_{x}=\left(V_{2}-V_{4}\right) / I_{1}\right]$ can be obtained by inverting the above matrix equation so that

$$
\left(\begin{array}{l}
V_{1} \\
V_{2} \\
V_{4}
\end{array}\right)=\left(\begin{array}{lll}
R_{11} & R_{12} & R_{14} \\
R_{21} & R_{22} & R_{24} \\
R_{41} & R_{42} & R_{44}
\end{array}\right) \cdot\left(\begin{array}{c}
I_{1} \\
I_{2} \\
I_{4}
\end{array}\right) .
$$

With $I_{2}=I_{4}=0$, we have

$$
\begin{aligned}
& V_{2}=R_{21} I_{1}, \\
& V_{4}=R_{41} I_{1} .
\end{aligned}
$$

From which it is seen that

$$
R_{x y}=\frac{V_{2}-V_{4}}{I_{1}}=R_{21}-R_{41},
$$

and

$$
R_{x x}=\frac{V_{1}}{I_{1}}=R_{11}
$$

The other two tensors $R_{y y}$ and $R_{y x}$ can obtained similarly by setting terminals 1 and 3 as the voltage probes with the electron wave passing from terminal 2 to terminal $4\left(I_{1}=I_{3}\right.$ $\left.=0, I_{2}=-I_{4}\right)$. Some simple algebra leads to

$$
R_{y x}=\frac{V_{1}}{I_{2}}=R_{12}-R_{13},
$$

and

$$
R_{y y}=\frac{V_{2}-V_{4}}{I_{2}}=\left(R_{22}-R_{23}\right)-\left(R_{32}-R_{33}\right) .
$$

Below we use these approaches to first evaluate the Hall effect in a homogeneous sample. That would set the stage for the subsequent consideration of quantum interference effect in patterned samples.

\section{MESOSCOPIC HALL EFFECT IN A HOMOGENEOUS SAMPLE}

We first evaluate, for a homogeneous square sample (with no scattering potential), the transmission coefficients for a single incident mode. The results are shown in Fig. 3. Here the size $a=200 \mathrm{~nm}$, Fermi wavelength $\lambda_{F}=12.28 \mathrm{~nm}\left(E_{F}\right.$ $=0.01 \mathrm{eV}$ ), and the magnetic field $B=0.1 \mathrm{~T}$. The grid number $N_{a}$ in this section is 200, which is large enough to achieve accurate results. There are a total of $32\left(\left[2 a / \lambda_{F}\right]\right.$ =32) guided modes. From the figure it is seen that with increasing mode index, $T_{13}$ decreases while $T_{12}$ and $T_{14}$ increase. Also the difference between $T_{12}$ and $T_{14}$ decreases for the larger mode index. Thus the transmission asymmetry induced by a magnetic field is larger for the low-lying modes than that for the high-lying modes. These relations can be explained semiclassically as follows.

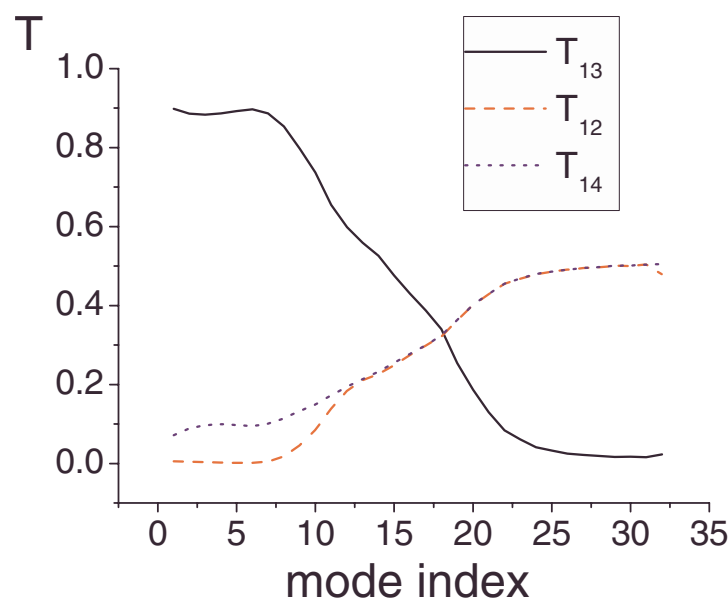

FIG. 3. (Color online) Transmission coefficients for different incident modes in a 32-mode square homogeneous sample ( $a$ $=200 \mathrm{~nm}, \lambda_{F}=12.28 \mathrm{~nm}$, and $B=0.1 \mathrm{~T}$ ).

The eigenmode in a $2 \mathrm{D}$ electron waveguide may be written as $e^{i k_{x} x} \sin (n \pi y / b)$. Here $k_{x}=\sqrt{k_{0}^{2}-(n \pi / b)^{2}}, \quad k_{0}^{2}$ $=2 m E_{F} / \hbar^{2}$, where $b$ is the waveguide width, $n$ is an integer for the mode index, and the waveguide axis is along the $x$ direction. The eigenfunction can be rewritten as $\left\{\exp \left[i k_{x} x\right.\right.$ $\left.+i(n \pi y / b)]-\exp \left[i k_{x} x-i(n \pi y / b)\right]\right\} / 2 i$ so that the mode may be viewed as the superposition of two plane waves with wave vectors $\left(k_{x}, n \pi / b\right)$ and $\left(k_{x},-n \pi / b\right)$. These two plane waves constitute two obliquely propagating "rays" that repeatedly reflect from the two sides of the waveguide [see Fig. 4(a1)]. This picture is identical to the ray model used in optical waveguides. ${ }^{9}$ At the open port of the waveguide, the two rays escape [Fig. 4(a2)], and for the low-lying mode with its smaller reflection angle [the ray's direction is determined by wave vector $\vec{k}:\left(k_{x}, n \pi / b\right)$ and $\left.\left(k_{x},-n \pi / b\right)\right]$, most of the wave is injected into the opposite terminal 3 [Fig. 4(b1)]. This implies a large $T_{13}$ and a small $T_{12}\left(T_{14}\right)$ (see Fig. $3)$. With a magnetic field, we can see that the electron flow injected into terminal $4\left(T_{14}\right)$ decreases and the electron flow into terminal $2\left(T_{12}\right)$ increases greatly [see Fig. 4(b2)]. This large change is due to the fact that both $T_{12}$ and $T_{14}$ are small when $B$ is zero so even a small percentage deviation from the large forward flow $\left(T_{13}\right)$ can greatly change the relative proportion between $T_{12}$ and $T_{14}$. With a similar analysis, we can see that for a high-lying mode with its larger boundary reflection angle most of the outgoing wave from terminal 1 would spread into terminal 2 or terminal 4 with equal likelihood [Fig. 4(c1)]. Only a little bit goes to terminal 3. This agrees with the large $T_{12}\left(T_{14}\right)$ value and a small $T_{13}$ as shown in Fig. 3. In a magnetic field that is not too large, the percentage change in $T_{12}$ and $T_{14}$ is small since most of the wave (rays) is already spread into terminals 2 and 4, and a small Lorentz force cannot alter that too much. This is made clear in Fig. 4(c2).

This semiclassical description omits some detailed interference effect that can lead to a much more complicated phenomenon in a four-terminal system. These rays can only approximately describe the motion of the electron waves and give a qualitative description for a large-sized system such as the 32-mode waveguide we just discussed. 


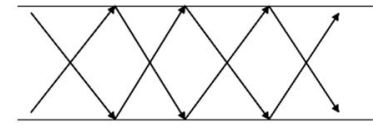

(a1)

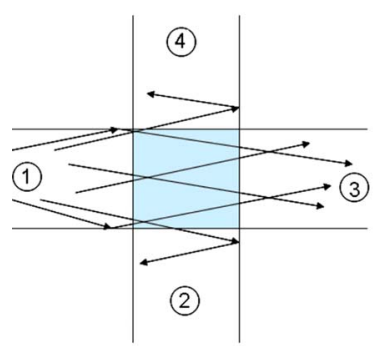

(b1)

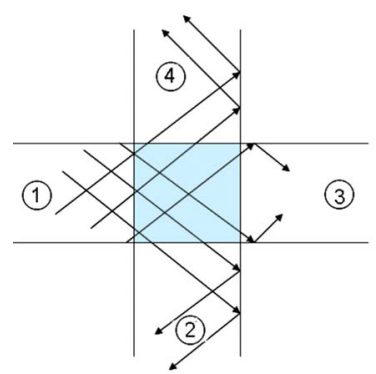

(c1)

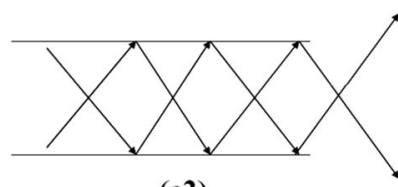

(a2)

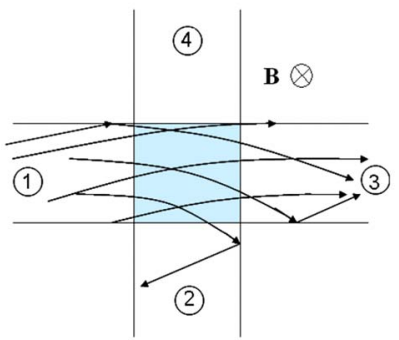

(b2)

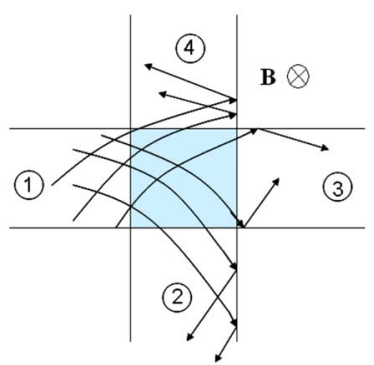

(c2)
FIG. 4. (Color online) Semiclassical (ballistic) description of mesoscopic electron transport in a four-terminal system. (a1) Two rightward propagating rays in a waveguide. (a2) The rays escape from the waveguide port. (b1) Low-lying mode with $B=0$. (b2) Low-lying mode under a magnetic field. (c1) High-lying mode with $B=0$. (c2) High-lying mode under a magnetic field.

When the sample size is as small as the electron's Fermi wavelength, the above semiclassical conclusion [that a lowlying mode has a large transmission difference $\left.\left(T_{12}-T_{14}\right)\right]$ is no longer always true. In such cases $T_{12}$ may be very close to $T_{14}$, or $T_{12}$ may even be less than $T_{14}$, which means Hall quenching or reverse Hall response (see the Hall coefficient in Fig. 5) can arise (from the wave interference effect). ${ }^{10}$

By using the approach described in Sec. II, we have calculated the Hall coefficient for a square sample with different sample size $(a)$ and a fixed Fermi wavelength $\left(\lambda_{F}\right)$. The results are shown in Fig. 5. Here $\lambda_{F}=78 \mathrm{~nm}$, corresponding to the electron density $n=1.03 \times 10^{11} \mathrm{~cm}^{-2}$. The magnetic field is $0.01 \mathrm{~T}$. The simulation results are noted to agree well with that in the literature..$^{10}$ In the figure we can see that when the $a / \lambda_{F}$ ratio is near $0.8, R_{H}$ is very close to zero. It can even be negative within a small range. This is the Hall quenching regime. When the sample size is small, $R_{H}$ exhibits an oscillatory behavior that eventually increases beyond the classical value when $a / \lambda_{F} \rightarrow 0.5$. When $a / \lambda_{F}$ is very large the oscillations tend to disappear with $R_{H}$ approaching an asymptotic constant, approximately the classical value $(n e)^{-1}$ indicated by the dashed line. There are cusps at $a / \lambda_{F}$ $=0.5,1.0,1.5, \ldots$ which result from the sudden appearance of an additional guided mode when $a / \lambda_{F}$ passes $N / 2(N$ is an integer)..$^{11}$

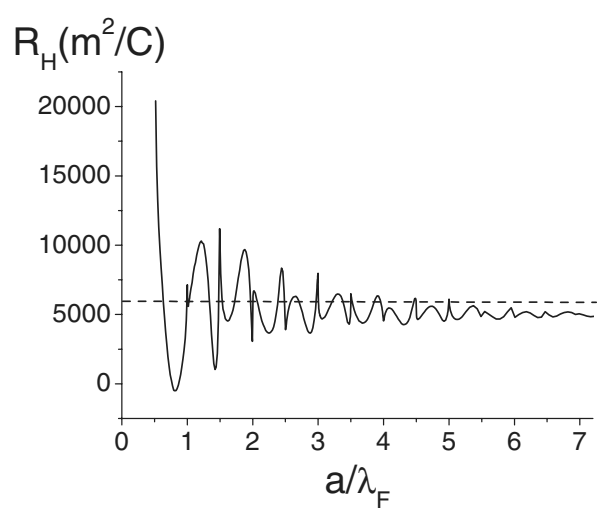

FIG. 5. Hall coefficient plotted as a function of $a / \lambda_{F}$ for a square mesoscopic sample. $\lambda_{F}$ is fixed at $78 \mathrm{~nm}$, corresponding to an electron density of $1.03 \times 10^{11} \mathrm{~cm}^{-2}$. The corresponding classical value of $R_{H}$ is $6.05 \times 10^{3} \mathrm{~m}^{2} / \mathrm{C}$, delineated by the dashed line.

\section{HALL EFFECT IN 2DEG WITH NANO- STRUCTURED PATTERNS}

From the results in the previous section we see that the size of a mesoscopic sample can influence the Hall coefficient. In this section we show that the quantum interference effect can significantly enhance the Hall effect by nanopatterning the sample when $a / \lambda_{F}$ is on the order of 1 .

\section{A. Quality factor $Q$}

To maximize the Hall effect, it is noted that $R_{H} \cdot B$ $=R_{x x} \cdot V_{H} / V_{0}$, where $V_{H}$ denotes the Hall voltage and $V_{o}$ the longitudinal voltage. A large $R_{H}$ can result from either a large $R_{x x}$ or a large $V_{H} / V_{0}$, or both. By focusing on the interference effect induced by the magnetic field to the leading order, we define a dimensionless quality factor $Q$ $=\left(V_{H} / V_{0}\right)(\widetilde{B})^{-1}$, where $\widetilde{B}$ is the dimensionless scaled magnetic field from Eq. (1), i.e., $\widetilde{B}=B \lambda_{F}^{2} / \phi_{0}$, where $\phi_{0}=h / e$ is the quantum magnetic flux. So the quality factor is

$$
Q=\left(V_{H} / V_{0}\right)\left(B \lambda_{F}^{2} / \phi_{0}\right)^{-1} .
$$

This $Q$ is invariant with respect to the simultaneous variation in sample size $(L \rightarrow K L)$ and the Fermi wavelength $\left(\lambda_{F}\right.$ $\left.\rightarrow K \lambda_{F}\right)$. This quality factor essentially discounts the Hallcoefficient variation due to the longitudinal resistance.

\section{B. Optimization with random selection}

Using this quality factor as our merit function, we attempt to maximize the $Q$ value with respect to the sample configuration in which certain regions are blocked (e.g., by a high potential $V$ ). We constrain our search to those patterns that are symmetric with respect to $y=a / 2$ (see Fig. 1), so as to ensure zero transverse voltage at zero magnetic field. In other words, this symmetric microstructure can ensure the offdiagonal terms in the resistivity tensor are antisymmetric (which vanish at zero magnetic field), without any symmetric off-diagonal components of the resistivity tensor. We show that for special sample configurations, the quality factor $Q$ can be increased significantly beyond the classical value. To 


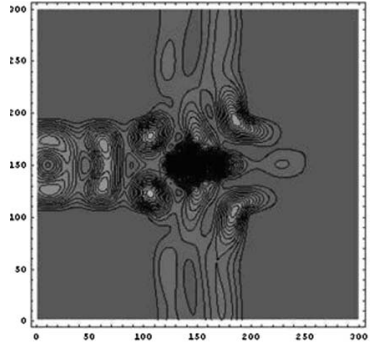

(a)

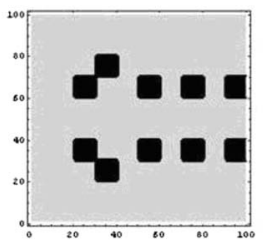

(c)

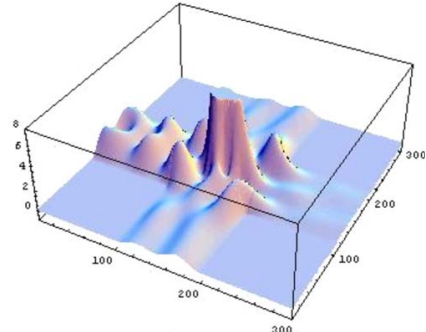

(b)

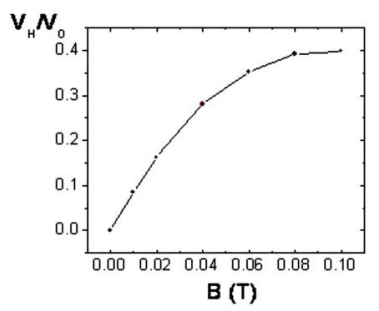

(d)
FIG. 6. (Color online) An optimal pattern from obtained from random selection. (a) The contour plot of the wave-function density. (b) The 3D plot of the wave-function density. (c) The optimal potential pattern (black blocks refer to the high potential regions). (d) $V_{H} / V_{0}$ dependence on the $B$ field. Here the sample size $a$ $=200 \mathrm{~nm}, \lambda_{F}=140.1 \mathrm{~nm}$, and $B=0.01 \mathrm{~T}$.

facilitate the search for such optimal configurations, we discretize the square sample into a 10 by 10 (or 20 by 20) grid. In each grid cell the potential can be either low $(V=0)$ or high (a large positive value for $V$ ). So the potential pattern can be represented by a $2 \mathrm{D}$ grid matrix, which can be further mapped into a $1 \mathrm{D}$ binary array. There are many ways to find the pattern (binary array) with an optimal $Q$ value. In this section we report the results where the patterns (binary arrays) were randomly generated. We have calculated $V_{H} / V_{0}$ (or $Q$ value) for each configuration and tried to isolate the one with the largest quality factor.

In the calculations, the sample size $a=200 \mathrm{~nm}$ and the Fermi wavelength is $140.1 \mathrm{~nm}$. For this Fermi wavelength and sample size in a homogeneous sample, $R_{x y}$ (or $Q$ value) is nearly zero, owing to the Hall quenching effect. For a small size of the obstacle (about $1 / 7$ of the Fermi wavelength) with a relatively low potential $\left(V=6.5 E_{F}\right)$, the tunneling effect has to be taken into account in the calculations. The grid number in the following calculations is 100 , which is large enough for numerical convergence. Figure 6 shows one of the optimized patterns, its wave-function distribution (without magnetic filed), and the $V_{H} / V_{0}$ dependence on the magnetic field $B$. With $B=0.01 \mathrm{~T}, V_{H} / V_{0}$ for this configuration is 0.085 . From the definition before, the $Q$ value is 1.78 . For the $V_{H} / V_{0}$ dependence shown in Fig. 6(d), the Hall voltage dependence is linear in $B$ in the limit of small magnetic field but displays a saturation behavior when $B$ is large.

It should be noted that the configuration with the high $Q$ value is very sensitive to small changes in its geometry. A small change in the position of one potential obstacle would decrease the $Q$ value significantly.

In Fig. 6 the wave-function density is noted to be high in the center of the sample. In other words, the electron wave is

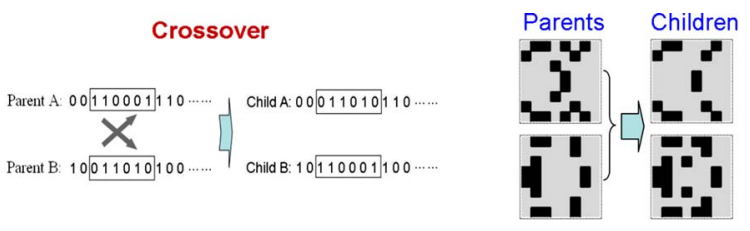

(a)

(b)

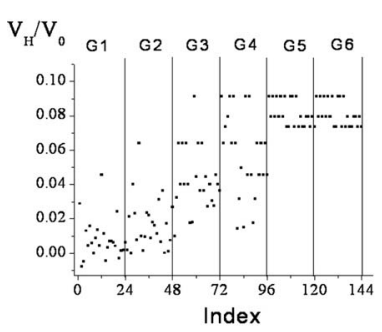

(c)

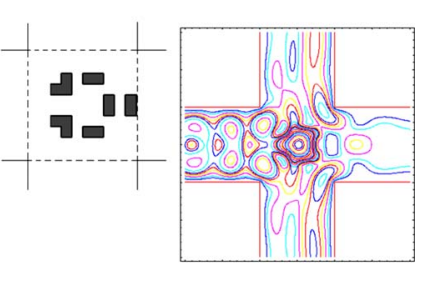

(d)
FIG. 7. (Color online) Genetic algorithm and the optimization process. (a) One of the reproduction procedures in GA: crossover. (b) The two parent patterns (left) and their children patterns (right) from crossover. (c) The results from one GA searching process. The parameter values are sample size $a=200 \mathrm{~nm}, \lambda_{F}=140.1 \mathrm{~nm}, B$ $=0.01 \mathrm{~T}$, grid number $N_{a}=100$, and potential $V=6.5 E_{F}$. (d) An optimized case from the GA search. The left panel is the potential pattern and the right panel shows the contour plot of the wavefunction density.

nearly localized in the sample. From a classical point of view we can explain why this can yield a high $Q$ value. When an electron wave is localized, the group velocity is very small. Also from the equation of motion for an electron in a magnetic field: $m v^{2} / R=q v B$, we can see that $R$ is sensitive to the velocity - a small velocity implies a small (bending) radius. This sensitivity makes the downward transmission $T_{12}$ (due to Lorentz force) much larger than the upward transmission $T_{14}$. From the Büttiker formula, $R_{x y}$ is proportional to $T_{12}$ $-T_{14}$. So such wave-function density distribution can lead to a large $R_{x y}$ and a large $Q$ value.

\section{Optimization with the genetic algorithm}

In order to accelerate the optimization process, we resort to the genetic algorithm (GA). ${ }^{12}$ This method originates from the analogy to biological evolution. A set of initial patterns (or the binary arrays) is generated randomly as the first generation. Each pattern is calculated for its $Q$ value and those with high $Q$ values are retained. The selected patterns would then "mate" with each other to "reproduce" their offspring patterns. Figure 7(a) shows such mating process. The two parent patterns have part of their gene codes (the strings in the frame) crossed over (or exchanged) to form the two children codes. These new codes are then mapped back to the 2D matrices for the children patterns, which is shown in Fig. 7(b). Besides crossover, there is also the mutation process, which means that some of the binary element changes from " 0 " to " 1 " or from " 1 " to " 0 ." Other types of mutation include the insertion or deletion of some part of the genetic code. Crossover can exchange some characteristics of their parents and transfer the new combinations of genetic infor- 


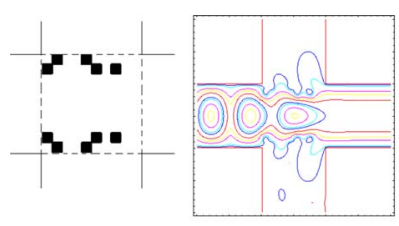

(a)

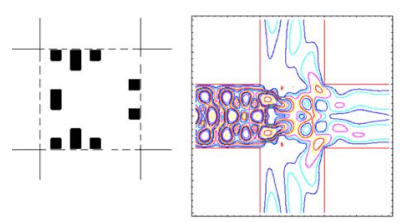

(c)

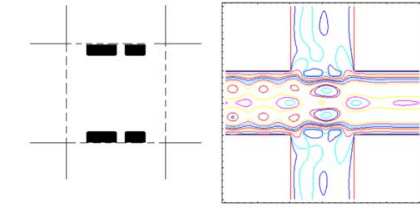

(b)

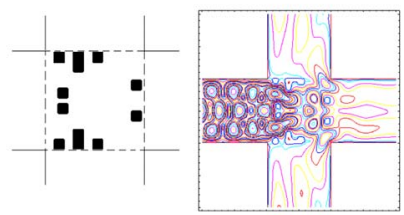

(d)
FIG. 8. (Color online) The optimal pattern (left panel) and the wave-function density contours (right panel). (a) One-mode case, $Q=3.40, \quad\left(a=75 \mathrm{~nm}, \lambda_{F}=78 \mathrm{~nm}\right)$. (b) Two-mode case, $Q=3.20$, $\left(a=111.4 \mathrm{~nm}, \lambda_{F}=78 \mathrm{~nm}\right)$. (c) Three-mode case, $Q=3.26$, $(a$ $\left.=150 \mathrm{~nm}, \lambda_{F}=78 \mathrm{~nm}\right)$. (d) Three modes case with a slightly modified pattern, $Q=4.75,\left(a=150 \mathrm{~nm}, \lambda_{F}=78 \mathrm{~nm}\right)$.

mation into their offspring patterns [compare the similarities between the parents and children patterns in Fig. 7(b)]. There is a high probability that some children patterns may share the "good" characteristics of both their parents so they may have better quality factors. In the next step, these good children patterns are selected as the parent patterns for the next generation. Such mate-selection procedures are repeated again and again until a high $Q$ value is achieved [see Fig. 7(c)]. The aim of mutation is to maintain genetic diversity by random perturbations. From Fig. 7(c) we see that when the generation number is large enough, the highest $Q$ values often cycle among several fixed values. These values may be in some local optimal regimes. Only with a larger searching scope in each generation (limited by the computation capacity) or proper mutation can this saturation phenomenon be effectively avoided.

Figure 7(d) shows one such optimal pattern obtained from a GA search with $a=200 \mathrm{~nm}, \lambda_{F}=140.1 \mathrm{~nm}$, and an obstacle potential $V_{0}=6.5 E_{F}$. It can be seen that the wave is again localized in the center of the sample. With $B=0.01 \mathrm{~T}$, we have $V_{H} / V_{0}=0.091$ and $Q=1.92$ for this optimal pattern.

We have also carried out a search using a much higher potential $\left(V=65 E_{F}\right.$, like a hard wall) for the obstacles. This is a more realistic choice since experimentally the high potential obstacles can be easily realized by etching holes in the semiconductor heterostructures. The electron density $n$ $=1.03 \times 10^{11} \mathrm{~cm}^{-2}$ was used in these calculations, with a corresponding Fermi wavelength $\lambda_{F}=78 \mathrm{~nm}$. Many GA search runs were carried out with 1-3 guided modes. Since the initial patterns in the GA search have an influence on the final optimal $Q$ values, a large number of patterns were initialized in each search run. Figure 8 shows the optimal patterns (left) and the corresponding wave-function density contours (right). Table I. gives a summary of the related parameter values for these optimal patterns.

It should be noted that in contrast to the optimal patterns in the previous cases [Fig. 6(a) and Fig. 7(d)], the first two optimal patterns here belong to the "side potential" type.
TABLE I. Parameter values for the optimal patterns shown in Fig. 8 (3* denotes the micro-optimized pattern based on sample 3$)$.

\begin{tabular}{|c|c|c|c|c|}
\hline Mode number & $\begin{array}{c}a \\
(\mathrm{~nm})\end{array}$ & $\begin{array}{c}\lambda_{F} \\
(\mathrm{~nm})\end{array}$ & $V_{H} / V_{0}(B=0.01 \mathrm{~T})$ & $Q$ \\
\hline 1 & 75.0 & 78.0 & 0.050 & 3.40 \\
\hline 2 & 111.4 & 78.0 & 0.047 & 3.20 \\
\hline 3 & 150.0 & 78.0 & 0.048 & 3.26 \\
\hline $3^{*}$ & 150.0 & 78.0 & 0.070 & 4.75 \\
\hline
\end{tabular}

Such potential patterns do not localize electron waves in the center. They pass most of the electron waves directly through the sample and only influence them at the two side regions. This is clearly another quantum interference strategy to achieve a large $V_{H} / V_{0}$ value.

When obstacles of such optimal patterns are slightly moved, $V_{H} / V_{0}$ may be further increased. In the three-mode case, the new pattern and the corresponding wave-function density contours are shown in Fig. 8(d). The voltage ratio here is 0.070 and $Q=4.75$. This modification from the pattern shown in Fig. 8(c) is equivalent to a small perturbation in a fine-grid configuration space around the optimal pattern. Due to our computational limit, we only searched a small range of such slight movements so as to give an example.

In the above we have predicted the optimal GHE patterns for single nanostructured patterns. It is entirely possible that assembling different nanopatterns into a macroscopic sample can lead to further enhancement. Such studies are left for future explorations.

\section{CONCLUDING REMARKS}

We have numerically evaluated the mesoscopic Hall effect for both homogeneous and nanostructured 2D samples. By using the genetic algorithm, we have obtained some optimal structures with large $Q$ values arising from the quantum interference effect. To give some perspective on the magnitudes of the effect, in Table II we present a comparison between the Hall parameters of metal, homogeneous heterostructure and our optimized nanostructured samples.

In Table II, both $R_{H}^{2 \mathrm{D}}$ and $R_{x x}$ for the silver sample are calculated classically. [ $R_{x x}$ is obtained from the Ohm's law, and $R_{x y}$ is obtained from the relation $R_{x y}=B \cdot R_{H}^{2 \mathrm{D}}$, with $R_{H}^{2 \mathrm{D}}$ $=1 /\left(n^{2 \mathrm{D}} e\right)$.] For the homogeneous semiconductor heterostructure and its nanostructured counterpart, $R_{x x}$ and $R_{H}^{2 \mathrm{D}}$ are obtained from the mesoscopic calculations. Since the $Q$ value is only used for mesoscopic calculations, case (1) does not have a $Q$ value. We can compare only the ratio $V_{H} / V_{0}$. It is seen that the metal case has a very much smaller $V_{H} / V_{0}$. It is also seen that $R_{H}$ for the patterned samples [Fig. 8(d)] can be up to $\sim 500 \%$ larger.

We note that while the enhancement factor in the present case is not too large, the absolute value of the Hall coefficient is orders of magnitude larger than the metallic system. As much of that difference arises from the difference in the resistivity of the difference material systems, it is proposed that the $V_{H} / V_{0}$ ratio, used in this work as the controlling 
TABLE II. Comparison of some Hall-effect-related quantities for different samples, all in the square geometry. The first row is for a silver sample with $a=1 \mathrm{~mm}, t=1 \mu \mathrm{m}, T=300 \mathrm{~K}$. The second row is for a semiconductor heterostructure with $n=1.0 \times 10^{11} \mathrm{~cm}^{-2}, a=150 \mathrm{~nm}, t=50 \mathrm{~nm}, T=10 \mathrm{~K}$. (It is a homogeneous sample). The third row is for the same semiconductor heterostructure as shown in the second row, but with optimized nanostructure pattern shown in Fig. 8(c), with $n=1.0 \times 10^{11} \mathrm{~cm}^{-2}, a=150 \mathrm{~nm}$, and $T$ $=10 \mathrm{~K}$

\begin{tabular}{lccccc}
\hline \hline Materials & $\begin{array}{c}n^{2 \mathrm{D}} \\
\left(\mathrm{cm}^{-2}\right)\end{array}$ & $\begin{array}{c}R_{H}^{2 \mathrm{D}} \\
\left(\mathrm{m}^{2} / \mathrm{C}\right)\end{array}$ & $\begin{array}{c}R_{x x} \\
(\mathrm{Ohm})\end{array}$ & $V_{H} / V_{0}(B=0.01 \mathrm{~T})$ & $Q$ \\
\hline $\mathrm{Ag}^{(1)}$ & $5.8 \times 10^{18}$ & $0.9 \times 10^{-4}$ & $1.6 \times 10^{-2}$ & $0.56 \times 10^{-4}$ & \\
$\mathrm{AlGaAs}^{-\mathrm{GaAs}^{(2)}}$ & $1.0 \times 10^{11}$ & $8.8 \times 10^{3}$ & $6.0 \times 10^{3}$ & 0.015 & 1.01 \\
GHE nanopattern $^{(3)}$ & $1.0 \times 10^{11}$ & $4.0 \times 10^{4}$ & $8.9 \times 10^{3}$ & 0.048 & 3.26 \\
\hline \hline
\end{tabular}

quality factor, should constitute a better unifying parameter to compare the Hall effect in different systems.

\section{ACKNOWLEDGMENTS}

We would like to thank X. X. Zhang, J. N. Wang, B. K. $\mathrm{Li}$, and L. Ding in the department of physics, HKUST for their helpful discussions. Partial financial support from the Research Grants Council of Hong Kong through Grant No. CA04/04.SC02 is gratefully acknowledged.

\section{APPENDIX A: EIGENMODES CALCULATION IN A WAVEGUIDE WITH MAGNETIC FIELD}

From Sec. II we know that for the waveguide solution $\psi=e^{i k \cdot x} \varphi(y)$ under a magnetic field [with Landau gauge $\vec{A}$ $=(B y, 0,0)], \varphi(y)$ satisfies the differential equation

$$
-\frac{d^{2} \varphi(y)}{d y^{2}}+\left[(k-P \cdot y)^{2}-k_{0}^{2}\right] \varphi(y)=0 .
$$

To obtain the eigenfunction $\varphi_{n}(y)$ under some specified boundary conditions, we use the eigenbasis expansion method. We choose the expansion basis as the sine functions $[\{\sin (n \pi y / a)\}$, with $a$ being the terminal width] because they naturally satisfy the boundary conditions $\varphi(0)=\varphi(a)=0$. Hence we write

$$
\varphi(y)=\sum_{n=1}^{N} F_{n} \sin \left(\frac{n \pi}{a} y\right) .
$$

By substituting Eq. (A2) into Eq. (A1) and making the derivative operation with some rearrangement, we obtain

$$
\begin{gathered}
P^{2} \sum_{n=1}^{N} F_{n} \cdot y^{2} \sin \left(\frac{n \pi}{a} y\right)-2 k P \sum_{n=1}^{N} F_{n} \cdot y \sin \left(\frac{n \pi}{a} y\right) \\
+\left[k^{2}-k_{0}^{2}+\left(\frac{n \pi}{a}\right)^{2}\right] \sum_{n=1}^{N} F_{n} \sin \left(\frac{n \pi}{a} y\right)=0 .
\end{gathered}
$$

Multiplying $\sin (m \pi y / a)$ on two sides of Eq. (A3) and integrating from 0 to $a$ yield

$$
\sum_{n=1}^{N} A_{m n} F_{n}=0,
$$

where

$$
A_{m n}=P^{2} \cdot P_{m n}-2 P k \cdot Q_{m n}+k^{2} \delta_{m n}+\left[k_{0}^{2}-(n \pi / a)^{2}\right] \delta_{m n},
$$

with

$$
P_{m n}=\frac{2}{a} \int_{0}^{a} y^{2} \sin \left(\frac{n \pi}{a} y\right) \sin \left(\frac{m \pi}{a} y\right) d y,
$$

and

$$
Q_{m n}=\frac{2}{a} \int_{0}^{a} y \sin \left(\frac{n \pi}{a} y\right) \sin \left(\frac{m \pi}{a} y\right) d y .
$$

In Eq. (A4) the matrix $\mathbf{A}$ is noted to contain the eigenvalue $k$. The matrices $P_{m n}$ and $Q_{m n}$ can be expressed analytically for specified $m$ and $n$. Only when the secular determinant $\operatorname{det}(\mathbf{A})$ is zero can there be nontrivial solutions of $\left\{F_{n}\right\}$, so as to ensure $\mathbf{A}(k) \mathbf{F}=\mathbf{0}$. This is the condition for finding the eigenvalue $k_{n}$. Since $\operatorname{det}(\mathbf{A})=\Pi_{i=1}^{N} \lambda_{i}$, where $\lambda_{i}$ is the eigenvalue of $\mathbf{A},{ }^{13}$ we need to calculate all the $\lambda_{i}$ and test if their product is zero for some $k$. Numerically, as $k$ passes through an eigenvalue, there is always one $\lambda_{i}$ which is very close to zero (in the real number case it changes sign). This condition is used to find the eigenvalue $k_{n}$. By substituting $k_{n}$ into $\mathbf{A}$ [Eq. (A4)], we obtain $\mathbf{F}$ and the eigenfunction $\varphi_{n}(y)$.

It should be noted that in the presence of a magnetic field the eigenvalues and eigenfunctions in the rightward propagating solution are not the same as those in the leftward propagating solution. We denote $k_{r}(n)$ and $\varphi_{n}(y)$ as the eigenvalue and eigenfunction, respectively, for the rightward propagating wave and $k_{f}(n)$ and $\varphi_{\bar{n}}(y)$ for the leftward propagating wave. Hence the eigensolutions in the two directions are $e^{i k_{r}(n) \cdot x} \varphi_{n}(y)$ and $e^{-i k_{f}(n) \cdot x} \varphi_{\bar{n}}(y)$.

In terminals 2 and 4 , the general solution is $\psi^{(2,4)}(x, y)$ $=e^{\mp i k \cdot y} \varphi(x)$. In order to separate the variables, another gauge $\vec{A}=(0,-B x, 0)$ has to be used in the Schrödinger equation. The differential equation for $\varphi(x)$ becomes [for $\psi^{(2)}(x, y)$ $\left.=e^{-i k \cdot y} \varphi(x)\right]$

$$
-\frac{d^{2} \varphi(x)}{d x^{2}}+\left[(k-P \cdot x)^{2}-k_{0}^{2}\right] \varphi(x)=0 .
$$

Equation (A5) is almost the same as Eq. (A1), only with $y$ replaced by $x$. We may be tempted to use the eigenfunction in Eq. (A1) [i.e., $\left.\varphi_{n}(y)\right]$ as the solution of Eq. (A5), and that would give 


$$
\psi^{(2)}(x, y)=e^{-i k_{r}(n) \cdot y} \varphi_{n}(x),
$$

which is wrong because the gauge here is not the same. We must use the gauge transformation to transform Eq. (A6) back to the previous gauge $(B y, 0,0)$ (Refs. 14 and 15),

$$
\begin{gathered}
\vec{A}_{1}=\vec{A}_{0}+\vec{\nabla} \Lambda, \\
\psi\left(\vec{A}_{1}\right)=\psi\left(\vec{A}_{0}\right) \cdot \exp \left(i \frac{e}{\hbar} \Lambda\right) .
\end{gathered}
$$

For our problem, $\psi\left(\vec{A}_{0}\right)$ is the wave function in Eq. (A6), $\vec{A}_{0}=(0,-B x, 0)$, and $\psi\left(\vec{A}_{1}\right)$ is the correct wave function in terminals 2 and 4 . Here $\vec{A}_{1}=(B y, 0,0)$ and $\vec{\nabla} \Lambda=(B y, B x, 0)$. So $\Lambda=B x y$, and Eq. (A8) can be written as

$$
\psi^{(2,4)}\left(\vec{A}_{1}\right)=\psi^{(2,4)}\left(\vec{A}_{0}\right) \cdot \exp \left(i \frac{e B}{\hbar} x y\right) .
$$

By substituting Eq. (A6) (with gauge $A_{0}$ ) into Eq. (A9), we obtain

$$
\psi^{(2)}\left(A_{1}\right)=e^{-i k_{r}(n) \cdot y} \varphi_{n}(x) \cdot \mathrm{e}^{i P x y},
$$

where $P=e B / \hbar$. For simplicity in calculation, we merge $\varphi_{n}(x)$ and $\exp (i P x y)$ in the above formula into a "new eigenfunction," denoted $\varphi_{\bar{n}}^{\prime}(x, y)$,

$$
\varphi_{\bar{n}}^{\prime}(x, y)=\varphi_{n}(x) \cdot e^{(i P \cdot x y)} .
$$

So the correct wave function in terminal 2 is

$$
\psi^{(2)}(x, y)=e^{-i k_{r}(n) \cdot y} \varphi_{\bar{n}}^{\prime}(x, y) .
$$

Similarly, we define a new eigenfunction $\varphi_{n}^{\prime}(x, y)$ in terminal 4 as

$$
\varphi_{n}^{\prime}(x, y)=\varphi_{\bar{n}}(x) \cdot e^{(i P \cdot x y)},
$$

and the wave function in terminal 4 is given by

$$
\psi^{(4)}(x, y)=e^{i k_{f}(n) \cdot(y-a)} \varphi_{n}^{\prime}(x, y) .
$$

Hence $e^{i k_{r}(n) \cdot x} \varphi_{n}(y), e^{-i k_{f}(n) \cdot(x-a)} \varphi_{\bar{n}}(y), e^{-i k_{r}(n) \cdot y} \varphi_{\bar{n}}^{\prime}(x, y)$, and $e^{i k_{f}(n) \cdot(y-a)} \varphi_{n}^{\prime}(x, y)$

are the eigenmode solutions in the four terminals.

\section{APPENDIX B: TRANSMISSION COEFFICIENT CALCULATION}

When the eigenmode expansion coefficients (like $\left\{A_{n}\right\}$ ) are obtained, we can calculate the transmission coefficients by starting from the flux of electron wave in the presence of a magnetic field, ${ }^{15}$

$$
\vec{j}=\frac{\hbar}{m_{e}} \operatorname{Im}\left[\psi^{*}\left(-\vec{\nabla}+\frac{i e}{\hbar} \vec{A}\right) \psi\right],
$$

where $m_{e}$ is the electron mass (to distinguish it from the index $m$ ). Here $\operatorname{Im}[]$ means the imaginary part of a complex number. For example, in terminal 3 , with $\vec{A}=(B y, 0,0)$, the $x$ component of the flux is given by

$$
j_{x}=\frac{\hbar}{2 m_{e}}\left[\psi^{*}\left(-i \partial_{x}-P y\right) \psi+\psi\left(i \partial_{x}-P y\right) \psi^{*}\right] .
$$

By substituting the corresponding solution in terminal 3, i.e., $\psi=\sum_{n}^{N_{1}} B_{n} \phi_{n}\left(\psi^{*}=\sum_{n}^{N_{1}} B_{n}^{*} \phi_{n}^{*}\right)$ into the above formula, we obtain

$$
j_{x}=j_{1}+j_{2},
$$

where

$$
\begin{gathered}
j_{1}=\frac{\hbar}{2 m_{e}} \sum_{n}^{M} \sum_{m}^{M} \phi_{n}^{*}\left(-i \partial_{x}-P y\right) \phi_{m} \cdot B_{n}^{*} B_{m}, \\
j_{2}=\frac{\hbar}{2 m_{e}} \sum_{n}^{M} \sum_{m}^{M} \phi_{n}\left(i \partial_{x}-P y\right) \phi_{m}^{*} \cdot B_{m} B_{n}^{*},
\end{gathered}
$$

and $\phi_{n}=\varphi_{n}(y) \cdot e^{i k_{r}(n) x}, M$ is the number of guided modes, and the evanescent modes have no contribution to the flux. By substituting the expression for $\phi_{n}$ into Eq. (B3) and carrying out the partial derivative, we obtain

$$
\begin{aligned}
j_{x}= & \frac{\hbar}{2 m_{e}} \sum_{n}^{M} \sum_{m}^{M} \varphi_{n}(y)\left[k_{r}(n)+k_{r}^{*}(m)\right. \\
& -2 P y] \varphi_{m}^{*}(y) \cdot B_{m} B_{n}^{*} e^{i\left[k_{r}(n)-k_{r}^{*}(m)\right] x} .
\end{aligned}
$$

Integration of $j_{x}$ from $y=0$ to $y=a$ yields the total flux. In the integration, the following orthogonal relation was used:

$$
\int_{0}^{a} \varphi_{n}(y)\left[k_{r}(n)+k_{r}^{*}(m)-2 P y\right] \varphi_{m}^{*}(y) \cdot d y=N_{p}(n) \delta_{n, m},
$$

where $N_{p}(n)\left[N_{q}(n)\right]$ denotes the normalization constant for the $n$th eigenfunction $\varphi_{n}(y)\left[\varphi_{\bar{n}}(y)\right]$ of Eq. (2). ${ }^{16}$ This relation can be obtained from the Sturm-Liouville theory. Finally, the total rightward flux is given by

$$
J_{x}^{\text {right }}=\frac{\hbar}{2 m_{e}} \sum_{n=1}^{M}\left|B_{n}\right|^{2} N_{p}(n) .
$$

Similarly, for the left-propagating eigenfunction $\varphi_{\bar{n}}(y)$, the orthogonal relation is given by

$$
\int_{0}^{a} \varphi_{\bar{n}}(y)\left[k_{f}(n)+k_{f}^{*}(m)+2 P y\right] \varphi_{\bar{n}}^{*}(y) \cdot d y=N_{q}(n) \delta_{n, m} .
$$

Also the total leftward flux may be expressed as

$$
J_{x}^{\text {left }}=\frac{\hbar}{2 m_{e}} \sum_{n=1}^{M}\left|A_{n}\right|^{2} N_{q}(n) .
$$

By using these results, it is easy to get the transmission and reflection coefficients for the rightward incident wave with mode $n$,

$$
T(n)=\sum_{m=1}^{M}\left|B_{m}^{n}\right|^{2} \frac{N_{p}(m)}{N_{p}(n)},
$$




$$
R(n)=\sum_{m=1}^{M}\left|A_{m}^{n}\right|^{2} \frac{N_{q}(m)}{N_{p}(n)} .
$$

In general, the transmission coefficient from port $i$ to port $j$ may be expressed as

$$
T_{i j}=\sum_{m}^{M} \sum_{n}^{M}\left|P_{n m}^{i j}\right|^{2} \cdot \frac{N_{p}(m)}{N_{p}(n)},
$$

where $P_{n m}^{i j}$ are the matrix elements coupling mode $n$ in the incident port $i$ to mode $m$ in port $j$.
${ }^{1}$ K. v. Klitzing, G. Dorda, and M. Pepper, Phys. Rev. Lett. 45, 494 (1980).

${ }^{2}$ A. B. Pakhomov, X. Yan, and B. Zhao, Appl. Phys. Lett. 67, 3497 (1995); A. B. Pakhomov and X. Yan, Solid State Commun. 99, 139 (1996).

${ }^{3}$ B. A. Aronzon, A. A. Likalter, V. V. Rylkov, A. K. Sarychev, M. A. Sedova, and A. E. Varfolomeev, Phys. Status Solidi B 205, 151 (1998); B. A. Aronzon, D. Y. Kovalev, A. N. Lagar'kov, E. Z. Meilikhov, V. V. Ryl'kov, M. A. Sedova, N. Nefre, M. Goiran, and J. Leotin, JETP Lett. 70, 90 (1999).

${ }^{4}$ X. X. Zhang, C. Wan, H. Liu, Z. Q. Li, P. Sheng, and J. J. Lin, Phys. Rev. Lett. 86, 5562 (2001).

${ }^{5}$ C. C. Wan and P. Sheng, Phys. Rev. B 66, 075309 (2002).

${ }^{6}$ S. Datta, Electronic Transport in Mesoscopic Systems (Cambridge University Press, Cambridge, 1995).

${ }^{7}$ H. Xie and P. Sheng, Phys. Rev. B (to be published).

${ }^{8} \mathrm{~A}$ free software package UMFPACK was used in solving the sparse matrix equation (http://www.cise.ufl.edu/research/sparse/ umfpack/).

${ }^{9}$ John David Jackson, Classical Electrodynamics, 3rd ed. (Wiley,
New York, 1999).

${ }^{10}$ H. U. Baranger and A. D. Stone, Phys. Rev. Lett. 63, 414 (1989); H. U. Baranger, D. P. DiVincenzo, R. A. Jalabert, and A. D. Stone, Phys. Rev. B 44, 10637 (1991).

${ }^{11}$ D. G. Ravenhall, H. W. Wyld, and R. L. Schult, Phys. Rev. Lett. 62, 1780 (1989).

${ }^{12} \mathrm{Z}$. Michalewicz, Genetic Algorithms+Data Structures =Evolution Programs (Springer, New York, 1999).

${ }^{13}$ From the property of determinant: $\operatorname{det}(\mathbf{A B})=\operatorname{det}(\mathbf{A}) \cdot \operatorname{det}(\mathbf{B})$, it is easy to see that $\operatorname{det}(\mathbf{A})=\operatorname{det}\left(\mathbf{G}^{-1} \cdot \boldsymbol{\Lambda} \cdot \mathbf{G}\right)$ $=\operatorname{det}\left(\mathbf{G}^{-1}\right) \cdot \operatorname{det}(\boldsymbol{\Lambda}) \cdot \operatorname{det}(\mathbf{G})=\operatorname{det}\left(\mathbf{G}^{-1} \mathbf{G}\right) \cdot \operatorname{det}(\boldsymbol{\Lambda})=\operatorname{det}(\boldsymbol{\Lambda})=\Pi_{i=1}^{N} \lambda_{i}$, where $\mathbf{G}$ is the square $N \times N$ matrix whose $i$ th column is the basis eigenvector $\mathbf{g}_{i}$ of $\mathbf{A}$, and $\boldsymbol{\Lambda}$ is the diagonal matrix whose diagonal elements are the corresponding eigenvalues, i.e., $\lambda_{i}$ $=\Lambda_{i i}$.

${ }^{14}$ R. L. Schult, H. W. Wyld, and D. G. Ravenhall, Phys. Rev. B 41, 12760 (1990).

${ }^{15}$ J. J. Sakurai, Quantum Mechanics (Addison-Wesley, Massachuestts, 1994).

${ }^{16}$ J. J. Palacios and C. Tejedor, Phys. Rev. B 48, 5386 (1993). 